\title{
A Situation Detection Mechanism for Pervasive Computing Infrastructures
}

\author{
Nikos Papageorgiou ${ }^{1}$, Dimitris Apostolou ${ }^{1,2}$, Yiannis Verginadis ${ }^{1}$, Andreas Tsagkaropoulos ${ }^{1}$, Gregoris Mentzas ${ }^{3}$ \\ ${ }^{1}$ National Technical University of Athens (NTUA), Athens, Greece \\ ${ }^{2}$ Department of Informatics, University of Piraeus, Piraeus, Greece
}

\section{\{npapag, jverg, atsagkaropoulos,gmentzas\}@mail.ntua.gr,dapost@unipi.gr}

\begin{abstract}
Pervasive computing infrastructures are highly dynamic and constant to continuous changes. Situation awareness in pervasive computing infrastructures involves the detection of situations requiring some kind of infrastructure adaptation or re-configuration. To realise situation awareness, the paper proposes an approach that is based on complex event processing. The associated software can process and analyse data streams generated both by the pervasive infrastructure as well as applications and services deployed on it. Moreover, the software is modular and can be easily deployed as a Docker container or a set of Docker containers. The software is independent of CEP libraries and we have shown that it can operate with both the Siddhi and Drools complex event processing libraries.
\end{abstract}

Keywords: Situation detection, pervasive computing, cloud, fog, edge

\section{INTRODUCTION}

Situation Awareness (SA) refers to the "perception of the elements in the environment within a volume of time and space, the comprehension of their meaning, and the projection of their status in the near future" [12], [13]. To realize systems for Situation Awareness, individual pieces of raw information (e.g. sensor data) should be interpreted into a higher, domainrelevant concept called situation, which is an abstract state of affairs interesting to specific applications. The power of using "situations" lies in their ability to provide a simple, humanunderstandable representation of, for instance, sensor data [23]. In the context of dynamic computing systems, situation is defined as an event occurrence that might require a reaction [1].

A situation is a set of contexts in the application over a period of time that affects future system behaviour. In pervasive computing, SA is the capability of the entities of the computing environment to be aware of situation changes and automatically adapt themselves to such changes to satisfy user requirements, including security and privacy [30]. SA is one of the most fundamental features to support dynamic adaptation of entities in pervasive computing environments.

A pervasive computing environment involves a set of cooperative entities, each of which has related context data. In order to support situation-aware adaptation of the entities in pervasive computing environments, it is necessary to model and specify context and situation in a way such that multiple entities can easily exchange, share and reuse their knowledge on context and situation [31], [32].

Pervasive computing infrastructures are highly dynamic and constant to continuous changes. For example, they may add or retract dynamically new computing nodes depending on the computing requirements of the application or services that are deployed on them. Hence, there is a need for facilitating situation awareness for detecting situations requiring some kind of infrastructure adaptation or re-configuration. To realise SA, specialised software should process and analyse data streams generated both by the pervasive infrastructure as well as applications and services deployed on it.

The scope of this work includes the design, development and evaluation SDM, a software component with capabilities to detect of situations with respect to the status of the pervasive infrastructure and deployment services and application. The goal of SDM is to identify interesting situations that might lead to the adaptation or reconfiguration of pervasive computing resources, such as edge devices, smartphones as well as fixed and cloud resources. The overarching objective of our work is to enhance pervasive computing infrastructures with capabilities to detect possible shortcomings and trigger corrective actions through issuing new adaptation recommendations.

\section{RELATED WORKS}

\section{A. Situation Modelling}

A situation is a subjective concept, whose definition depends on sensors in a current system, which decide available contexts used in a specification; on the environment where the system works, which determines the domain knowledge to be applied (e.g., a spatial map); and on the requirement of applications, which determines what states of affairs are interesting. The same sensor data can be interpreted to different situations according to the requirements of applications [33]. Situations are composite entities whose constituents are other entities, their properties and the relations in which they are involved [9]. Situations support us in conceptualizing certain "parts of reality that can be comprehended as a whole" [18].

Situations are often reified (such as in [4], [9]), or ascribed an "object" status [20], which enables one not only to identify situations in facts but also to refer to the properties of situations themselves. For example, we could refer to the duration of a particular situation or whether a situation is current or past, which would enable us to say that the situation "Drone out of range" occurred yesterday and lasted two hours. The temporal aspect of situations also enables us to refer to change in time, thus we could say that "VM1's CPU 
utilisation is rising" or that "VM2 memory has been overdrawn for the last 15 days".

Costa, et al. [8] developed the Situation Modeling Language (SML) which is a graphical language for situation modeling, allowing the expression of primitive situation types and complex situation types (with temporal constraints when required) SML allows composition of situations using the temporal formal relations defined by Allen [2], namely before, meets, overlaps, starts, during, finishes, coincides and their relations (after, met by, overlapped by, started by, includes, and finished by).

A situation type definition in SML is a composition of two kinds of models [29]: a context model and a situation type model. The context model is a structural model that defines the classes of entities and relationships that exist in the modelled domain, which in turn are referred by the situation type model entities. In order to define context models, SML employs an ontologically well-founded UML class diagram profile called OntoUML [17].

In Context Toolkit [34], situation is modelled on a system level as the aggregation of context, but there is no language level situation modelling. Situation calculus and its extensions [26] model situation based on the effects of actions and events, and consider situation as a complete state of the world. A core SAW ontology [25] models situation as a collection of Goals, SituationObjects and Relations using UML, and can be converted to OWL representation. A conceptual model for context and situation for service-based systems and a situation specification example based on the conceptual model using Flogic are presented in [31].

Kalyan et al. [19] introduced a multi-level situation theory, where an intermediate level micro situation is introduced between infons and situations. Infons and situations are two of the major conceptual notions in situation theory. An infon embodies a discrete unit of information for a single entity (e.g., a resource node), while a situation makes certain infons factual and thus support facts. Situations are considered as a hierarchical aggregation of micro situations and situations. This work aims to assist information reuse and support ease of retrieving the right kind of information by providing appropriate abstraction of information. Using the concept of micro situations, the authors address how to handle complex user queries by creating simpler entity-specific micro situations and further combining them to arrive at users' current situation and as well as to enable efficient reasoning. We follow a similar approach in which we allow for different abstraction levels for situations, as described in the following section.

\section{B. Situation Detection}

Unlike other ICT domains, situations in computing infrastructures are highly related to sensor data, domain knowledge on environments and applications. Sensor data occur in large volumes, in different modalities, and are highly inter-dependent, dynamic and uncertain. Situations are in a rich structural and temporal relationship, and they evolve in diffuse boundaries. In addition, the complexity in domain knowledge and applications makes studying situations a very challenging task. [33].

Specification-based approaches represent expert knowledge in logic rules and apply reasoning engines to infer proper situations from current sensor input [33]. Existing approaches range from earlier attempts in first-order logic [28] to more advanced logic models that aims to support efficient reasoning while keeping expressive power, see, e.g., Loke 2010 [24]. With their powerful representation and reasoning capabilities, ontologies have been widely applied, see Chen et al. 2004 [5]. As more and more sensors are deployed in real-world environments for a long term experiment, the uncertainty of sensor data starts gaining attention. To deal with the uncertainty, traditional logic-based techniques need to be incorporated with other probabilistic techniques [11]. Specification-based approaches have introduced uncertainty metrics to describe sensor data, including incompleteness, accuracy, timeliness, and reliability [15], [22], [7]. The concept hierarchy in ontologies are typically used to evaluate the precision of sensor data against the conditions of rules.

Machine learning techniques have been widely applied to learning complex associations between situations and sensor data [33]. Most of the examined learning techniques are supervised learning, such as naïve Bayes, Bayesian networks, HMMs, CRFs, and so on. These techniques learn the models and parameters from training data that usually requires a human to label a situation to sensor data that are observed during the occurrence of this situation. When there exists a large number of situations to be identified, manual labelling of training data may place a significant burden on developers involved in the data collection. Therefore, supervised learning techniques may have limitations in real-life deployment where scalability, applicability, and adaptability are highly concerned [16]. To tackle this issue, researchers have employed unsupervised learning approaches. Among them, suffix-tree and Jeffrey divergence can extract features from sensor observations, which are distinguishable from one situation to another [33]. A neural network is classified as unsupervised, although parameters of neural networks can sometimes be estimated using supervised learning. Web mining techniques are not strictly unsupervised learning in that they perform the learning on web documents, rather than on the collected sensor data.

Compared to specification-based approaches, a distinguishable features of the learning-based approaches is their ability in uncovering a pattern or correlation underlying data. Learning-based approaches can be used to extract categorical features from numerical sensor data; for example, learning network surges or abnormal VM power consumption from sensor data. They can learn correlations between a combination of relevant categorical or numerical sensor data and situations; for example, learning the pattern of how services consume memory resources when they perform a certain activity or run a specific method.

Learning-based approaches have a stronger capability to resolve uncertainty by training with the real-world data that involves noise. These approaches not only learn associations 
between sensor data and situations, but also the effect that the uncertainty of sensor data has on the associations. For example, the conditional probabilities learned in naïve Bayes includes the reliability of sensor data as well as the contribution of the characterised sensor data in identifying a situation [33].

\section{SituATION MODEL \& APPROACH}

\section{A. Situation Model}

We follow an event-based approach for situation modelling and detection. We consider sensor data or event encompassing raw (or minimally processed) data retrieved from both physical sensors and 'virtual' sensors observing systems, services and applications such as network traffic. These data are used to form context - the environment in which the system operates, and situations, which are considered as an abstraction of the events occurring in the real world.

We define a situation as an external semantic interpretation of events. Interpretation means that situations assign meanings to events; external means that the interpretation is performed from the perspective of applications, rather than from events; semantic means that the interpretation assigns meaning on events based on structures and relationships within the same type of events and between different types of events [33]. A situation can uncover meaningful correlations between events, labelling them with a descriptive name. The descriptive name can be called a descriptive definition of a situation, which is about how a human defines a state of affairs in reality.
In Figure 1, we describe the proposed Situation Metamodel that captures the concepts and artefacts based on which the SDM will be able to detect meaningful situations. Such situations may reveal impending failures or even opportunities for increasing the performance of the deployed Big Data intensive applications over multi-cloud and edge resources. Although, metamodeling refers to the analysis and development of a number of rules and constraints, applicable for modelling a predefined class of problems, here we use the term Metamodel to describe the frame of concepts and their associations that should be followed for implementing SDM's core capabilities.

A Situation may comprise AtomicSituation and CompositeSituation. An AtomicSituation represents any basic situation whose value is directly derived from the value of a ComplexEvent. A ComplexEvent is composed of SimpleEvents (e.g. raw incoming events) and expresses a ScalabilityRequirement (e.g. if RAM $>80 \%$ and CPU $>60 \%$ for at least 5 minutes...) that should drive the Adaptation of the big data intensive application according to a ScalabilityAction (e.g. ... then scale horizontally).

The CompositeSituation represents complicated situations pertained to the logical composition and temporal composition of AtomicSituations. The logical composition over other situations refer to the ConjunctionSituation (i.e. combining two or more AtomicSituations using the logical AND operator), DisjunctionSituation (i.e. combining two or more AtomicSituations using the logical OR operator), and NegationSituation (i.e. combining two or more

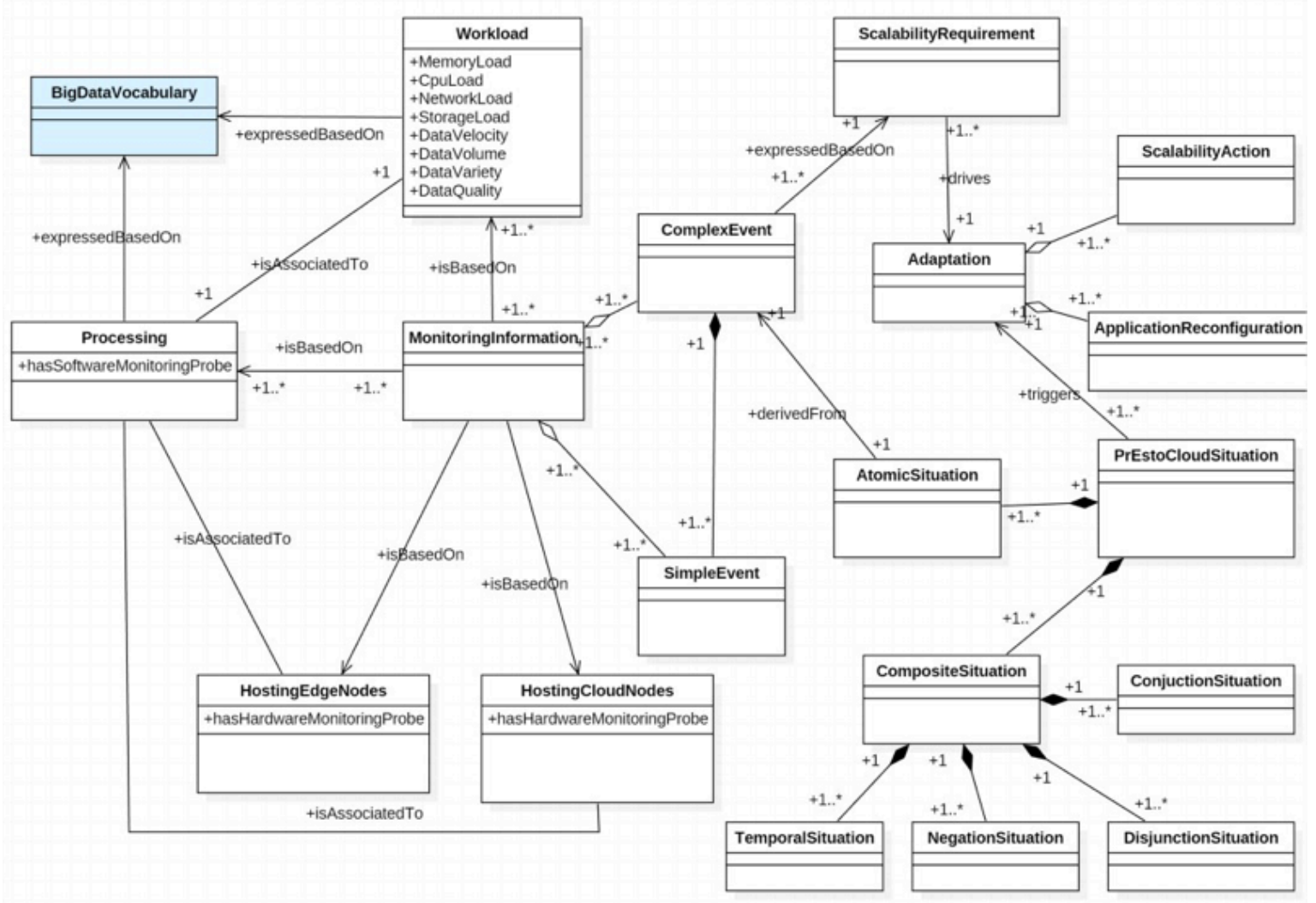

Figure 1. Situation Metamodel 
AtomicSituations using the logical NOT operator); the temporal composition can be implemented using the TemporalSituation that describes certain time-related dependencies or sequence associations between two or more AtomicSituations. A situation may occur before, or after another situation, or interleave with another situation.

A CompositeSituation can be decomposed into a set of smaller situations, which is a typical composition relation between situations. For example, a 'Cold VM migrating' situation is composed of a 'Relocating configuration and storage files' situation, a 'Moving VM to new host' situation and a 'Powering off VM' situation. According to our metamodel aggregating SimpleEvents and ComplexEvents we acquire the related MonitoringInformation which is necessary for checking the health status and QoS of both the deployed big data intensive application and the underlying multi-cloud and edge resources. Thus, all the MonitoringInformation is based on the Processing, HostingEdgeNodes, HostingCloudNodes and current Workload detected through the appropriate software, hardware and workload related monitoring probes, respectively. Both Processing and Workload are expressed based on the BigDataVocabulary in order to abstractly map types of big-data streams to big data processing services types revealing their importance for the detected situations. The BigDataVocabulary refers to an external class that includes all the concepts and properties to be used for describing Big Data characteristics that should be considered for making Big Data application placement decisions.

\section{B. Situation Detection Approach}

In industry, cloud platforms that support automatic or semiautomatic adaptation use event driven rules in order to decide the time of adaptation. Amazon AWS, for example, provides auto-scaling services [3] that trigger adaptation actions based on user-configurable rules that are evaluated in real-time using internal or external monitoring infrastructure. Kubernetes [21] provides auto-scaling capabilities based on internal or external metrics. In Google Cloud [14], users can specify a target CPU utilisation for a group of (service) instances, the platform will try to maintain it by scaling it up or down. OpenStack [27] also supports auto-scaling policies by deploying the Heat service. Autoscaling in OpenstackHeat is triggered by Alarms produced by the telemetry service (Ceilometer).

Since, pervasive computing combines multi-cloud and edge resources, we need a mechanism to detect situations from heterogeneous devices and services with very different capabilities in terms of computational resources and provide the ability to control and customize the execution environment. For example edge devices may have very low computational resources or a very restricted (due to security reasons) environment for custom applications. Very often these devices have low network bandwidth, unpredictable disconnections from the network and data transmission spikes that are caused by external events (such as social events, weather conditions or other). In this environment we need mechanisms for data-driven event detection. Therefore, we opted for an approach that relies on complex event processing technologies, which are capable of processing in real-time a large number of events generated by a variety of distributed cloud and edge computing resources as well as other data generating sensors. A complex event is an event derived from a group of events using either aggregation of derivation functions. Information enclosed in a set of related events can be represented (i.e., summarized) through such a complex event.

Arguably, situation detection in a pervasive computing environment needs to take care of network bandwidth consumption. Similarly, to commercial systems, it is important to support parts of the situation detection at the edge. For example Cisco routers with Cisco IOS ${ }^{\circledR}$ XE [6] are able to run $\mathrm{KVM}$ virtual machines or LXC containers. It is crucial to have low computation resource consumption (memory and CPU) and ability to efficiently distribute and process events in multiple stages.

\section{ARCHITECTURE \& IMPLEMENTATION}

\section{A. Situation Detection Mechanism Architecture}

Figure 2 depicts the conceptual architecture of SDM. Data from the pervasive infrastructure (physical and virtual machines, containers, applications, services, etc. ) and edge devices (mobile phones, IoT devices) are published as events to the Broker in specific topics. One or more SDM service instances subscribe to the desired topics and receive streams of events that contain up-to-date information about the current state of those entities (e.g. used RAM, CPU consumption, disk $\mathrm{I} / \mathrm{O}$, requests per second, etc.).

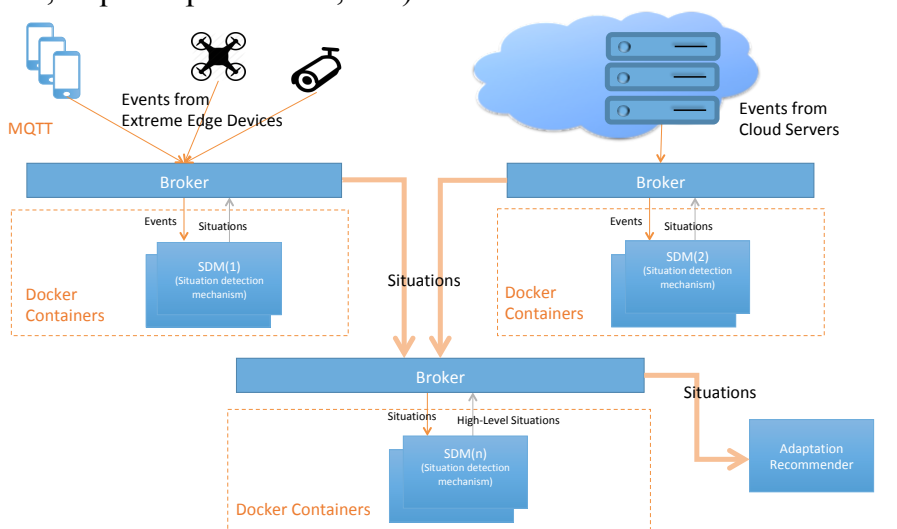

Figure 2. Situation Detection Mechanism Architecture

The SDM instances process these events based on the supplied CEP rules which are defined in order to detect interesting situations. Several SDM instances can be used in parallel or in series in order to process the incoming event streams. High level situations can be detected by processing low-level situations from many SDM instances.

\section{B. Implementation}

The Situation Detection Mechanism has been developed in Java. It can be deployed as a set of Docker containers. SDM receives events from the Communication and Message Broker, which is implemented by a RabbitMQ container. A Logstash Docker container can be used for the preprocessing of the 
incoming events. Logstash can subscribe to RabbitMQ topics, process incoming events, and publish the events back to RabbitMQ (in different topics). Some very common tasks assigned to Logstash included the transformation of the event payload (from CSV to JSON, or from an initial JSON format to a JSON format with additional fields) and the mapping of different event types to new topics based on which were extracted from the event payload. Logstash can be used also as an input interface that can support additional messaging protocols like UDP, Graphite, UDP, XMPP or Beats with different input plugins (https://www.elastic.co/guide/en/logstash/current/inputplugins.html).

After pre-processing, the input events are published through RabbitMQ to a Docker container that embeds and runs a CEP engine. We demonstrate that the implementation of the proposed approach can be agnostic to the CEP engine used. There are many CEP libraries that can be used at this stage with SDM such as Drools, WSO2 Siddhi and Esper. The basic functional requirements from the CEP Docker container include the a) ability to read all the necessary configuration (input parameters and topics, output parameters and topics, rules) from files or environment variables, b) the ability to consume events from RabbitMQ in JSON format, c) the ability to produce new events that denote the detection of a situation, d) the ability to publish events to RabbitMQ in JSON format, d) the ability to read, process, and produce different JSON event formats dynamically by changing only the rule file (without the need for example to write and compile new code in Java or any other programming language in order to create new event models).

It may be very critical for the selected CEP library to present the lowest possible computing resource consumption, latency or footprint as well as the maximum throughput. This depends on the environment that a CEP container is deployed (cloud, edge, extreme edge), the type of situations that it will be instructed to detect, and the expected input workload (in terms of events per second). Important factors for the selection of a CEP library are also the expressivity of the supported rule description language and the complexity from the user's point of view for the implementation of the required CEP patterns.

We have selected and compared two different CEP libraries, the WSO2 Siddhi 4 and the Drools CEP engine. Both are distributed with open source licenses. Drools is a widely used rule engine and CEP library. Siddhhi on the other hand is newer and is reported to offer state of the art performance when compared to other libraries including Esper [10].

\section{Evaluation}

In this section we evaluate and compare the Drools- and Siddhi-based implementations of SDM. The hardware used is: A KVM Virtual Machine with 4 cores and 8GB RAM running on a server with Intel Xeon E7@ 2.4 Ghz CPU. Software used includes: SDM services run under Ubuntu 17.10 with the following software packages installed: Docker version 17.12.0-ce, build c97c6d6, Docker-compose version 1.19.0, build 9e633ef , OpenJDK Runtime Environment (build
1.8.0 171-8u171-b11-0ubuntu0.17.10.1-b11), 3.7.5 (Docker image rabbitmq3.7.5-management).

The RabbitMQ load-testing tool is used to generate and publish events (https://github.com/rabbitmq/rabbitmq-perftest) to SDM services. With PerfTest we can select the number of event producers, the length of the period that we want to send events, the frequency with which the event producers should generate events and the payload of the events (from a list of files). The AMQP exchange name and the topic are also configurable.

With a Java Management Extensions (JMX) tool such as JConsole or VisualVM (https://visualvm.github.io/) we monitor many metrics of Java applications. We use JMX to monitor the Drools and Siddhi version of SDM. We run (with docker-compose) one Siddhi CEP engine and one Drools CEP engine in parallel and configure them to subscribe to the same AMQP exchange and topic. In this way both CEP engines receive the same events from PerfTest.

The payload of the events is a JSON file that contains different values of two attributes named "memory" and "cpu", (without any timestamp for simplification reasons) like the following:

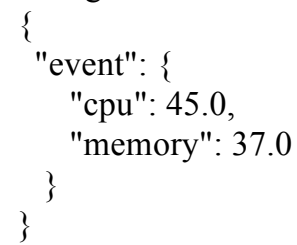

Both Drools and Siddhi were configured to produce every 10 sec two events containing: the average CPU and MEMORY (during the last 10s), the number of MEMORY and CPU events that it received (during the last 10s).

First we run PerfTest for 60 seconds with increasing number of event producers that send one event per second.

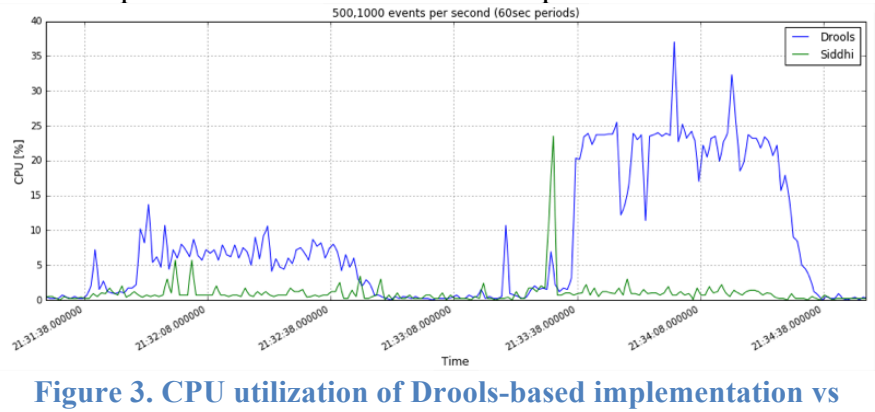
Siddhi-based implementation $(500,1000$ events/sec)

In Figure 3, we compare the CPU consumption of Drools and Siddhi while sending 500 events per second and 1000 events per second, for two consecutive periods of 60 seconds. We can clearly see that the Siddhi-based implementation of SDM has much lower total CPU utilization than the Drools-based implementation which increases in a bigger proportion as the rate of incoming events increases. 


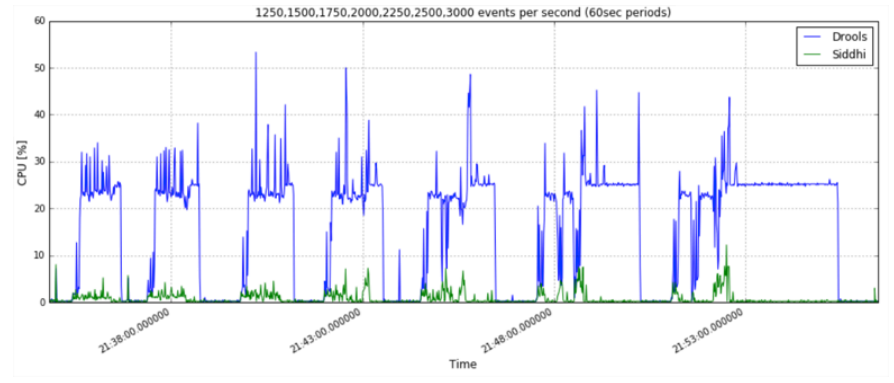

Figure 4. CPU utilization of Drools-based implementation vs Siddhi-based implementation (1250 to 3000 events/sec)

In Figure 4, we continue the same experiment with increasing number of events per second (generated by PerfTest) : 1250, 1500, 1750, 2000, 2250, 3000. It is again clear that Siddhi has much lower CPU utilisation. It is also notable that after 1500 events per second the Drools-based implementation of SDM queues the incoming messages and continues processing an increasing number of seconds after PerfTest has finished sending events. Siddhi processes all the events in almost real-time in the above tests.

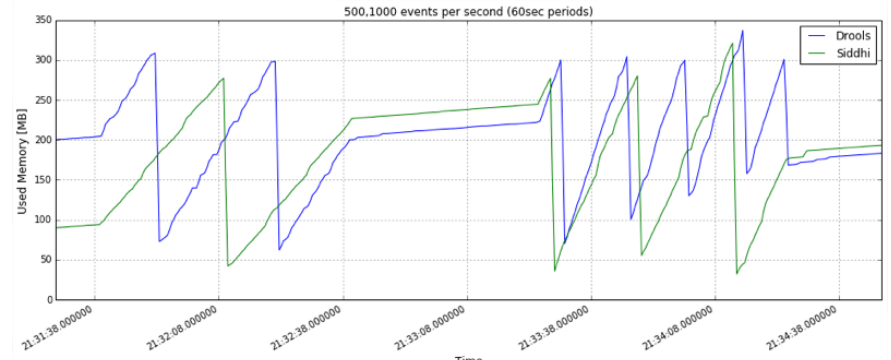

Figure 5. Used memory of Drools-based implementation vs Siddhi-based implementation $(500,1000$ events/sec)

In Figure 5, we can see the memory consumption of Drools and Siddhi when sending 500 events per second and 1000 events per second. In these event rates, both CEP engines have similar memory consumption.

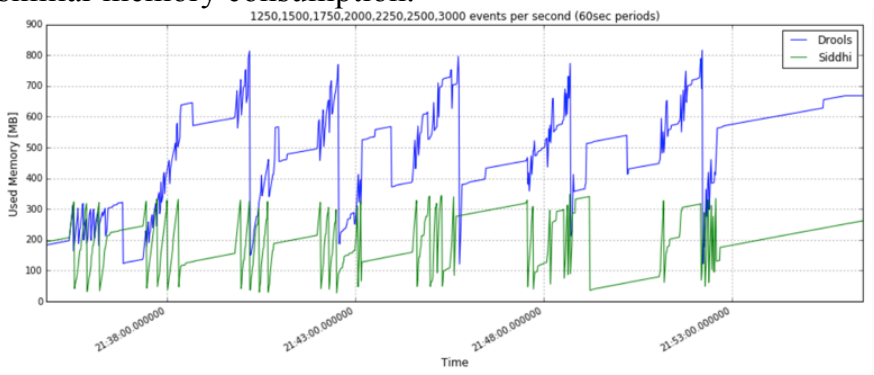

Figure 6. Used memory of Drools-based implementation vs Siddhi-based implementation (1250 to 3000 events/sec)

In Figure 6, we can see the memory consumption of Drools and Siddhi when sending in range from 1250 to 3000 events per second, in consecutive 60 second periods. After 1500 events per second Drools needs more memory than Siddhi (the peak of difference is about $500 \mathrm{MB}$ ). In the following two diagramms we present in the left axis the CPU consumption and in the right axis the memory consumption of Drools during the tests that we described before.

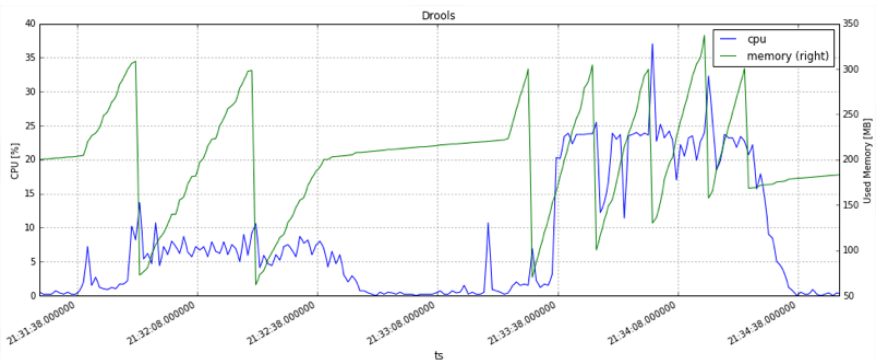

Figure 7. Drools-based implementation CPU utilization (blue) and used memory (green) $(500,1000$ events/sec)

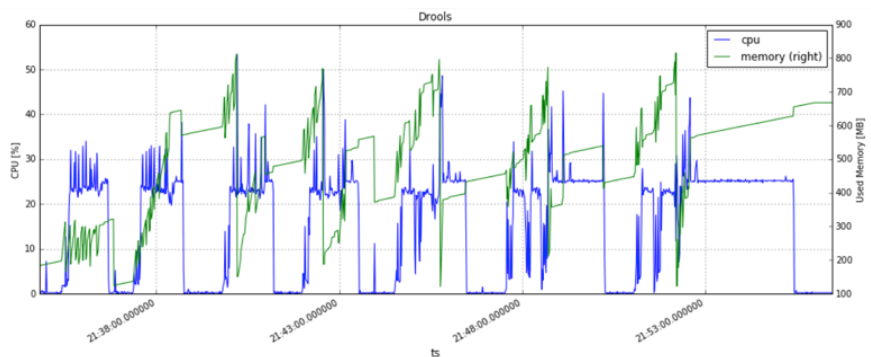

Figure 8. Drools-based implementation CPU utilization (blue) and used memory (green) (1250 to 3000 events/sec)

Figure 9 depicts the queued messages in RabbitMQ (red colour) and the message rates (in yellow the publish rate and in green the deliver rate). We can observe that RabbitMQ was queuing many messages above 3000 evens per second rate.

Queued messages last hour ?

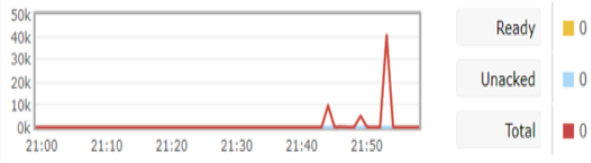

Message rates last hour ?

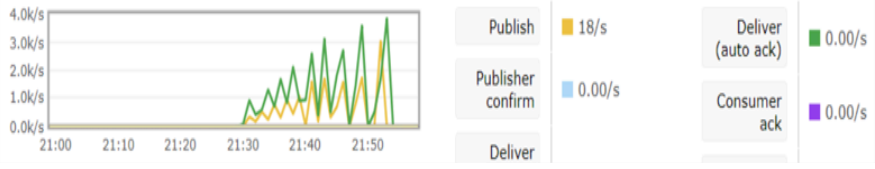

Figure 9. RabbitMQ management console metrics during SDM load testing with PerfTest (consecutive 60s period tests with increasing rates from 500 to 3000 events per second)

If we test Drools and Siddhi for bigger time periods, over 1500 events per second we can see clearly in the following diagrams that Drools takes much more time to process the incoming events. These diagrams have been produced by sending 1500 events per second for 5 minutes $(300 \mathrm{sec})$ to Siddhi and Drools with PerfTest. 


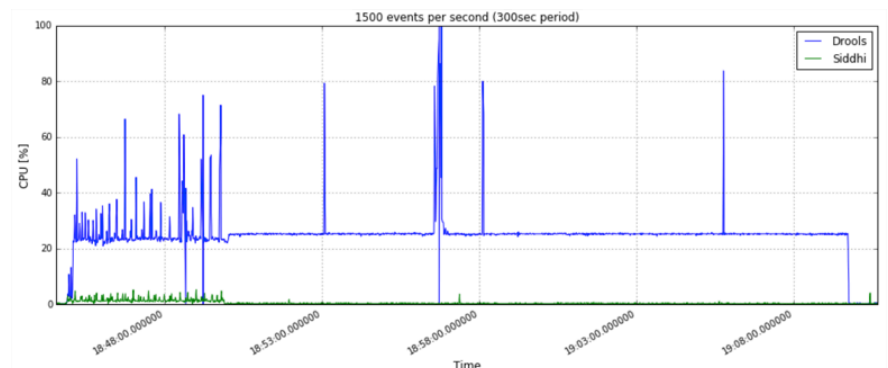

Figure 10. CPU utilization of Drools-based implementation vs Siddhi-based implementation (sending 1500 events/sec for 5 minutes)

Figure 10 depicts the CPU consumption of the two implementations of SDM while load-testing the with 1500 events/sec for of a period of 300 seconds. From the CPU consumption diagrams we can confirm that the Drools-based implementation of SDM still processes the data 20 minutes after PerfTest has completed sending events.

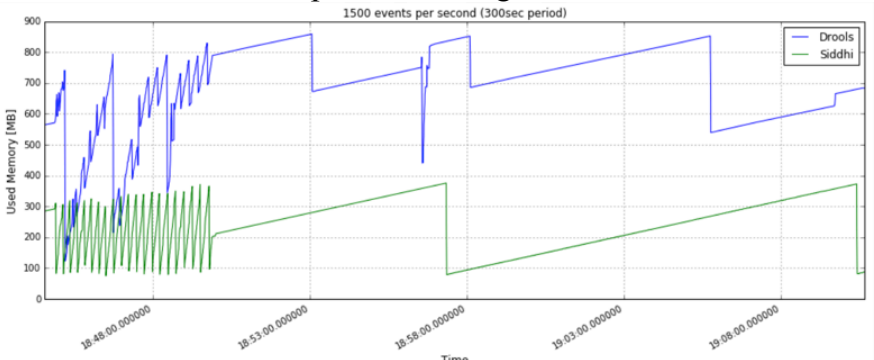

Figure 11. Used memory of Drools-based implementation vs Siddhi-based implementation (sending 1500 events/sec for 5 minutes)

Figure 11 depicts the memory consumption of the two implementations of SDM while load-testing the with 1500 events/sec for of a period of 300 seconds.

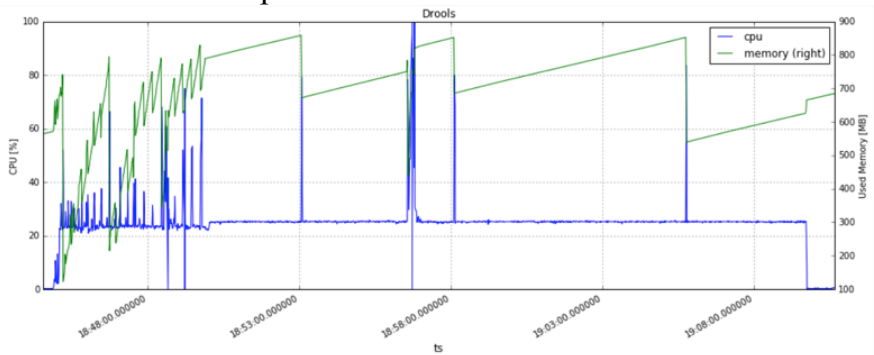

Figure 12. CPU utilization vs used memory of Drools-based implementation (sending 1500 events/sec for 5 minutes)

Figure 12 depicts in the same diagram the CPU utilization and the memory consumption of the Drools-based implementation of SDM while load-testing it with 1500 events/sec for of a period of 300 seconds.

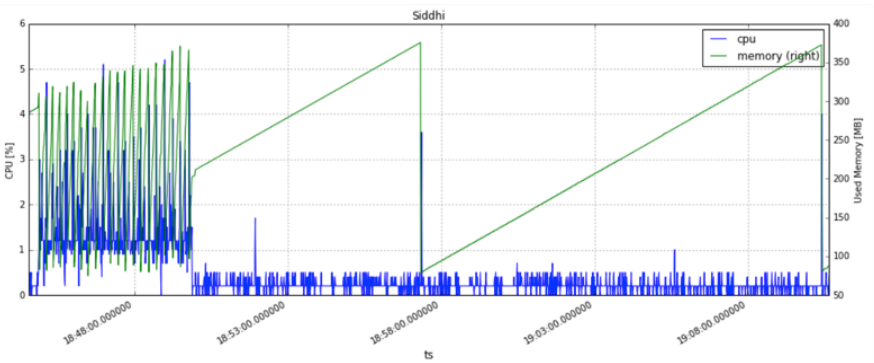

Figure 13. CPU utilization vs used memory of Siddhi-based implementation (sending 1500 events/sec for 5 minutes)

Figure 13 depicts in the same diagram the CPU utilization and the memory consumption of the Siddhi-based implementation of SDM while load-testing it with 1500 events/sec for of a period of 300 seconds. In contrast with the Drools-based implementation Figure 12 CPU utilization falls to much lower than $1 \%$ after PerfTest has finished sending events.
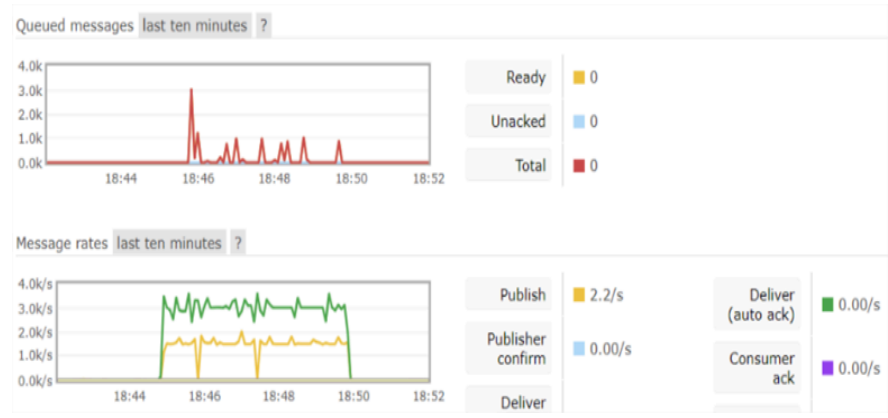

Figure 14. RabbitMQ management console metrics during SDM load testing with PerfTest (5min period)

The diagrams in Figure 14 are produced by the RabbitMQ management console. The diagram named "Queued messages" depict the amount of queued messages in RabbitMQ during the load-testing of the system with PerfTest for 5 minutes (or 300 seconds). In the diagram named "Message rates" the green line represents the deliver message rate while the yellow line represents the publish message rates. As we can see the publish message rate is very close to 1500 events/sec (as we instructed PerfTest to do). The deliver message rate is twice because we have two subscribers (the Drools-based and the Siddhi-based instances of SDM).

\section{CONCLUSIONS}

This paper presented Situation Detection Mechanism, a software component which allows the detection of situations that require some kind of pervasive infrastructure or application adaptation. SDM does so by processing and analysing data streams generated by data-intensive applications and services deployed on pervasive computing resources such as computing resources at the extreme edge of the network.

We designed the SDM component so as it is modular and can be easily deployed as a Docker container or a set of Docker containers. Moreover, we designed SDM to be independent of CEP libraries and we have shown that it can operate with both the Siddhi and Drools CEP libraries. Testing 
and evaluation of SDM revealed that it is capable to detect situations defined as complex event patterns. Specifically, we tested SDM in conjunction with both Drools and Siddhi in two scenarios: first, we stress-tested it using the PerfTest loadtesting tool of RabbitMQ. Tests indicated that SDM can be used to detect situations expressed as complex event patterns. Moreover, out tests have shown that Siddhi can scale better than Drools.

Our work focused on providing detection capabilities for situations that are few and can be modelled manually. Hence, we followed a specification-based approach. Our future work will evaluate the specification-based approach and, if needed, we will augment it with learning-based methods and techniques to cope with more complex situations, which cannot be manually specified, as well as with imperfect sensors.

\section{ACKNOWLEDGMENT}

This work is partly funded by the European Commission project H2020 PRESTOCLOUD (732339).

\section{REFERENCES}

[1] Adi, A. and O. Etzion, "Amit - the situation manager", The VLDB Journal, vol. 13, no. 2, pp. 177-203, May 2004.

[2] Allen, J. F. "Maintaining knowledge about temporal intervals," Communications of the ACM, vol. 26, Nov. 1983, pp. 832-843.

[3] Amazon (2018), https://aws.amazon.com/autoscaling.

[4] Barwise, J., The Situation In Logic, CSLI Lecture Notes 17, 1989.

[5] Chen, H., T. Finin, A. Joshi, An ontology for context-aware pervasive computing environments, Knowledge Engineering Review 18 (3) (2004) 197-207. Special Issue on Ontologies for Distributed Systems.

[6] Cisco https://www.cisco.com/c/dam/en/us/products/collateral/routers/asr-1000series-aggregation-services-routers/q-and-a-c67-737653.pdf.

[7] Cohen, N.H., H.Lei, P.CastroII, J.S.D,A.Purakayastha. Composing pervasive data using iQL, in: WMCSA'02: Proceedings of the Fourth IEEE Workshop on Mobile Computing Systems and Applications, 2002, pp. 94-104.

[8] Costa, et al. "A model-driven approach to situations: Situation modeling and rule-based situation detection." Enterprise Distributed Object Computing Conference (EDOC), 2012 IEEE 16th International. IEEE, 2012.

[9] Costa, P. D., G. Guizzardi, J.P.A. Almeida, L. Ferreira Pires, M. van Sinderen, "Situations in Conceptual Modeling of Context". Workshop on Vocabularies, Ontologies, and Rules for the Enterprise (VORTE 2006) at IEEE EDOC 2006, IEEE Computer Society Press, 2006.

[10] Dayarathna, M., \& Perera, S. (2018). Recent Advancements in Event Processing. ACM Computing Surveys (CSUR), 51(2), 33.

[11] Delir, P., Haghighi, S. Krishnaswamy, A. Zaslavsky, M.M. Gaber, Reasoning about context in uncertain pervasive computing environments, in: EuroSSC'08: Proceedings of the 3rd European Conference on Smart Sensing and Context, Springer-Verlag, Berlin, Heidelberg, 2008, pp. 112-125.

[12] Endsley, M. Designing for Situation Awareness: An Approach to UserCentered Design, Second Edition. CRC Press, 2016.

[13] Franke, U. and J. Brynielsson, "Cyber situational awareness - A systematic review of the literature", Computers \& Security, vol. 46, pp. 18-31, 2014.

[14] Google Cloud https://cloud.google.com/compute/docs/autoscaler/.

(2018),

[15] Gray, P.D., D. Salber, Modelling and using sensed context information in the design of interactive applications, in: EHCl'01: Proceedings of the 8th IFIP International Conference on Engineering for Human-Computer Interaction, Springer-Verlag, London, UK, 2001, pp. 317-336.

[16] Gu, T., S. Chen, X. Tao, J. Lu. A nons upervised approach to activity recognition and segmentation based on object-use fingerprints, Data and Knowledge Engineering 69 (6) (2010) 533-544.

[17] Guizzardi, G. "Ontological foundations for structural conceptual models," CTIT, Centre for Telematics and Information Technology, Enschede, 2005.

[18] Hoehndorf, R. "Situoid theory, An ontological approach to situation theory", M.Sc. Thesis, University of Leipzig 2005.

[19] Kalyan, A., Gopalan, S., \& Sridhar, V. (2005, March). Hybrid context model based on multilevel situation theory and ontology for contact centers. In Pervasive Computing and Communications Workshops, 2005. PerCom 2005 Workshops. Third IEEE International Conference on (pp. 3-7). IEEE.

[20] Kokar, M. M., C. J. Matheus and K. Baclawski, "Ontology-based situation awareness," Information Fusion, vol. 10, Jan, 2009, pp. 83- 98.

[21] Kubernetes (2018), https://kubernetes.io/docs/tasks/runapplication/horizontal-pod-autoscale-walkthrough/.

[22] Lei, H., D.M. Sow, S. John, I. Davis, G. Banavar, M.R. Ebling, The design and applications of a context service, SIGMOBILE Mobile Computing and Communications Review 6 (4) (2002) 45-55.

[23] Loia, V., G. D'Aniello, A. Gaeta, and F. Orciuoli, "Enforcing situation awareness with granular computing: A systematic overview and new perspectives", Granular Computing, vol. 1, no. 2, pp. 127-143, 2016.

[24] Loke, S.W. Incremental awareness and compositionality: a design philosophy for context-aware pervasive systems, Pervasive and Mobile Computing 6 (2) (2010) 239-253.

[25] Matheus, C. J., M. M. Kokar, and K. Baclawski, "A Core Ontology for Situation Awareness", Proc. 6th Int'l Conf. on Information Fusion, 2003, pp. $545-552$.

[26] McCarthy, J. "Situation Calculus with Concurrent Events and Narrative", http://wwwformal.stanford.edu/jmc/narrative/ narrative.html, 2000.

[27] OpenStack (2018), https://docs.openstack.org/senlin/latest/scenarios/autoscaling_heat.html.

[28] Ranganathan, A., J. Al-Muhtadi, R.H. Campbell, Reasoning about uncertain contexts in pervasive computing environments, IEEE Pervasive Computing 03 (2) (2004) 62-70.

[29] Sobral, V. M., Almeida, J. P. A., \& Costa, P. D. (2015, March). Assessing situation models with a lightweight formal method. In Cognitive Methods in Situation Awareness and Decision Support (CogSIMA), 2015 IEEE International Inter-Disciplinary Conference on (pp. 42-48). IEEE.

[30] Yau, S. S., \& Liu, J. (2006, April). Hierarchical situation modeling and reasoning for pervasive computing. In Software Technologies for Future Embedded and Ubiquitous Systems, 2006 and the 2006 Second International Workshop on Collaborative Computing, Integration, and Assurance. SEUS 2006/WCCIA 2006. The Fourth IEEE Workshop on (pp. 6-pp). IEEE.

[31] Yau, S. S., Huang, D., Gong, H., \& Yao, Y. (2006). Support for situation awareness in trustworthy ubiquitous computing application software. Software: Practice and Experience, 36(9), 893-921.

[32] Yau, S. S., Y. Wang, and F. Karim, "Development of Situation-Aware Application Software for Ubiquitous Computing Environments", Proc. 26th Ann. Int'l Computer Software and Applications Conf., 2002, pp. 233-238.

[33] Ye, J., Dobson, S., \& McKeever, S. (2012). Situation identification techniques in pervasive computing: A review. Pervasive and mobile computing, 8(1), 36-66.

[34] Dey, A. K., \& Abowd, G. D. (2000, June). The context toolkit: Aiding the development of context-aware applications. In Workshop on Software Engineering for wearable and pervasive computing (pp. 431441). 